\title{
L'enveloppe solaire : un retour vers le futur
}

\section{Solar Envelope: A return to the future}

\author{
Laila Koubaa Turki ${ }^{1, *}$, Khaoula Raboudi ${ }^{1}$ et Abdelkader Ben Saci ${ }^{2}$ \\ ${ }^{1}$ Univ. Carthage, ENAU, Equipe de recherche M2A, Sidi Bou Saïd, Tunisie \\ ${ }^{2}$ Univ. Grenoble Alpes, CNRS, ENSAG, AAU-CRESSON, 38000 Grenoble, France
}

Résumé. Cet article propose un retour réflexif sur le modèle de l'enveloppe solaire. Il s'agit d'un regard rétrospectif des approches numériques de ce modèle qui se voulait dès le départ servir l'activité de conception. Le développement des modèles de calcul énergétique a permis d'affiner la détermination de ses contraintes. L'avancée numérique a donné lieu à des enveloppes dérivées intégrant, en plus de l'accès solaire, des paramètres physiques ou réglementaires. Cependant, l'avancée numérique de l'enveloppe solaire n'est pas utilisée pour répondre aux défis climatiques actuels. Ce papier propose de dresser un état de l'art de l'enveloppe solaire, de ses développements et de ses applications en vue de comprendre en quoi la promesse des années soixante-dix est aujourd'hui réaliste. C'est l'occasion aussi de discuter le rôle que joue le progrès scientifique et technique de la morphose numérique dans les pratiques architecturales et urbaines.

Mots-clés. Accès solaire, Enveloppe solaire, Méthodes de générations, Morphologie, culture numérique.

\begin{abstract}
This paper proposes a reflexive feedback to the solar envelope's model. It is a retrospective examination of this model digital approaches of which was intended from the outset to serve the design activity. The development of energy calculation models made possible to refine its constraints determination. The digital advance has given rise to derived envelopes integrating, in addition to solar access, physical or regulatory parameters. However, reality shows that the solar envelope's digital progress is not meeting the current climatic challenges. This paper proposes to draw up a review of the solar envelope evolution in order to understand how the promise of the seventies is now achievable. It is also an opportunity to discuss the role of the scientific and technical progress of digital morphosis in architectural and urban practices.
\end{abstract}

Keywords. Solar access, Solar envelope, Generation methods Morphology, Digital culture.

\footnotetext{
* Corresponding author: koubaa.leila@yahoo.fr
} 


\section{Introduction}

Dans le contexte actuel de la transition énergétique et numérique du bâtiment, il est évident d'intégrer l'énergie solaire à la conception architecturale et urbaine. L'optimisation de la morphologie des bâtiments est un levier pour diminuer la consommation d'énergie ; notamment en assurant l'accès solaire des bâtisses avoisinantes. L'économie d'énergie en architecture est un enjeu majeur depuis la crise pétrolière des années soixante-dix. De nombreux mouvements sont apparus ; architecture bioclimatique, architecture verte, etc., qui prônaient la réduction de l'utilisation des énergies fossiles. Cet engouement a perdu son ampleur dès le début des années quatre-vingts à cause de la disponibilité et la baisse du prix du baril de pétrole. Aujourd'hui, quarante ans plus tard, il y a un retour, d'une manière franche, à une situation de crise qui s'amplifie avec le réchauffement climatique.

De nombreux travaux ont porté sur la morphologie permettant de préserver l'accès solaire des constructions [1-6]. L'évolution numérique a accompagné cette problématique aussi bien sur le plan des méthodes et des algorithmes que celui de la capacité des machines pour la simulation solaire et les calculs énergétiques.

Parmi ces travaux, le concept de l'enveloppe solaire décrit par Knowles entre 1968 et 1971 [2, 7] était promoteur et a connu un effort de formalisation, de modélisation et de développement numérique. Il proposait un concept morphologique qui voulait garantir le droit solaire ; permettre l'accès solaire à un bâtiment sans ombrager les constructions voisines. Ce concept serait aujourd'hui inopérant sans numérique. Comment le numérique a participé alors à son évolution?

Cet article propose de dresser un inventaire réflexif des différents travaux ${ }^{2}$ de recherche portant sur l'enveloppe solaire afin de comprendre l'implication du numérique dans le développement de ce concept et ses méthodes de génération. Ce modèle est-il encore d'actualité ? Dans quel sens devrait-il encore évoluer pour répondre aux attentes actuelles et futures ? Il s'agit ici d'un retour vers le futur afin d'interroger les développements à venir et d'en esquisser des projections.

\section{Culture numérique et enveloppe solaire : retour vers le futur}

L'enveloppe solaire décrit les limites volumétriques qui n'ombragent pas le voisinage à des moments spécifiques [7]. Ces moments appelés le temps de coupure (cut-off-time), définissent la période pour laquelle l'accès solaire est souhaitable. L'ombrage est inscrit dans une limite, appelée la clôture d'ombre (Shadow fences), qui définit la frontière de projection de l'ombre de la volumétrie. Le temps de coupure et la clôture d'ombre sont les deux contraintes de définition morphologique de l'enveloppe solaire.

L'examen des travaux fondés sur ce concept permet de proposer quatre groupes de lecture. Un premier groupe de travaux a utilisé l'enveloppe solaire pour évaluer l'accès solaire et étudier son impact. Favorisé par l'essor du numérique, un deuxième groupe de travaux s'est intéressé aux méthodes de génération numérique de l'enveloppe solaire. Un troisième groupe a étudié et affiné la détermination de ses contraintes. Enfin, un dernier groupe, a proposé des modèles d'enveloppes dérivées qui intègrent, en plus de l'accès solaire, d'autres contraintes urbaines ou physiques. Cette répartition est ici conceptuelle. En effet, les préoccupations des travaux de ces groupes ne sont pas étanches les unes aux autres.

\footnotetext{
${ }^{2}$ Les publications sont ici des marqueurs des travaux de recherche sur l'enveloppe solaire.
} 


\subsection{Usage de l'enveloppe solaire}

Plusieurs travaux ont utilisé l'enveloppe solaire comme un dispositif d'évaluation de l'accès solaire afin de vérifier si une construction enfreint le droit solaire [8-14]. D'autres travaux ont étudié son impact sur d'autres facteurs de conception tels que la densité urbaine obtenue [12, 15-20]; la consommation énergétique [21]; le captage solaire [22, 23] et le confort thermique extérieur [24] et intérieur [25].

\subsection{Méthodes de génération de l'enveloppe solaire}

La construction géométrique de l'enveloppe solaire est une tâche complexe, en particulier pour des parcelles avec des limites irrégulières ou sur un sol en pente. Les premières méthodes utilisaient la géométrie de l'ensoleillement (trajectoire solaire, angle d'obstruction). Les calculs se faisaient manuellement en utilisant les données de l'azimut et de l'élévation solaire aux heures retenues aux solstices d'hiver et d'été sans calculer la quantité d'énergie associée. La génération de l'enveloppe solaire se basait sur des méthodes graphiques puis en 1986 Vonderohe et al. [26] en ont donné une formalisation mathématique. Le développement des outils numériques à partir des années 90 a donné lieu à des méthodes de génération semi-automatique [27-30] à l'aide des logiciels de DAO et à des méthodes de génération automatique utilisant un codage informatique. Ces derniers ont donné lieu à des logiciels/applications dédiés. Nous citons SolVelope [31], CalcSolar [32], SustARC [33], SolCAD [34], PIRAMIDA [35], SVR [36] et CityZoom [37].

Depuis l'avènement des outils de conception paramétrique et de programmation visuelle, le nombre de publications qui proposent des composants paramétriques de génération de l'enveloppe solaire a augmenté. Elle est alors devenue facile à calculer, à visualiser et à manipuler. La programmation visuelle est accessible aux architectes car elle ne demande pas beaucoup de connaissances en codage. De plus, la modélisation paramétrique donne la possibilité d'agir interactivement sur les différents paramètres servant à sa génération. Plusieurs composants paramétriques ont vu le jour. Nous citons le composant enveloppe solaire sous API Revit [38]; les composants utilisés sous Grasshopper Ladybug Solar Envelope [39], SolarEnvelopeAdvanced [40], DIVA for Rhino [41], enveloppe solaire de Capeluto et al. [25] et enveloppe solaire de Santiago [23] ; le plugin T4SU utilisé sous SketchUp [42]; enfin le module de l'enveloppe solaire sous Dynamo [43].

Les outils numériques de génération de l'enveloppe solaire se basent aujourd'hui sur six méthodes. 1- Calculer une série de profils de coupe de l'enveloppe solaire en fonction de la direction des rayons solaires et du temps d'accès solaire [28, 44, 45]. 2- Calculer l'altimétrie des sommets d'un maillage de la parcelle de sorte à permettre l'accès solaire au voisinage [25, 34, 40, 46-48]. 3- Extruder les faces d'un maillage pour atteindre ce même objectif [49]. 4- Générer l'enveloppe solaire en inversant la projection de l'ombre en représentation raster de l'élévation numérique urbaine ${ }^{3}[50,51]$. 5- Découper le volume d'extrusion verticale de la parcelle en fonction de la direction des rayons solaires et la clôture d'ombre. Ceci peut être fait par une opération booléenne d'intersection entre le volume extrudé, selon la direction solaire, du polygone de la clôture d'ombre et celui de la parcelle $[21,29,36,37,39,52]$ ou la découpe de ce dernier par des plans définis par les arrêtes de la clôture d'ombre et la direction des rayons solaires [38, 42]. 6- Générer des volumes élémentaires dits voxel sous contrainte de l'accès solaire à un voisinage. La

\footnotetext{
${ }^{3}$ Modèle numérique d'élévation urbaine consiste à représenter l'information 3D en image raster selon une échelle de niveau de gris proportionnelle à la hauteur du bâti.
} 
visibilité des voxels à partir de ce voisinage en direction du soleil procède par addition ou par soustraction de voxels sur la parcelle [53, 54].

\subsection{Méthodes de détermination des contraintes de l'enveloppe solaire}

Une première contrainte est le temps de coupure déterminé selon les périodes d'insolation utile ou de captage d'énergie solaire. Knowles [44] a proposé de calculer les périodes utiles d'accès solaire par la pondération du rayonnement solaire, incident à plusieurs moments de la journée, par le sinus de l'angle de l'altitude du soleil.

L'avancée des modèles de calcul énergétique notamment Radiance [55] et Energyplus [56] ont permis d'affiner la détermination du temps de coupure. Capeluto et al. [17] ont décrit une méthode qui se base sur la quantité d'irradiance solaire requise pour chaque orientation. Les chercheurs ont proposé les profils de coupe qui représentent l'angle du soleil le plus bas pour la période requise. Ils ont proposé de définir le temps de coupure selon les positions solaires [25]. La méthode consiste à filtrer les rayons solaires dont l'impact est insignifiant en fonction de l'angle d'incidence. Raboudi et al. [57] ont utilisé l'outil de calcul Info-Récepteur Solaire ${ }^{4}$ pour déterminer les heures d'accès solaire souhaitables. Les chercheurs ont calculé l'irradiance solaire cumulative (en $\mathrm{W} / \mathrm{m}^{2}$ ) reçue par heure et par orientation de façades au solstice d'hiver. Vartholomaios [58] a suivi une approche similaire en délimitant le temps de coupure en fonction des gains solaires moyens reçus par heure pendant la saison froide pour différentes orientations par rapport aux besoins de chauffage. La date de référence de l'ensoleillement est déterminée graphiquement en traçant sur le diagramme solaire un masque d'ombrage. Koubaa Turki et al. [59] se sont basés sur la loi Pareto ${ }^{5}$. Les heures retenues correspondent à $20 \%$ des heures de la journée et qui permettent $80 \%$ de l'irradiance directe reçue par les façades au solstice d'hiver. Bruce [60] a défini le temps de coupure en fonction des besoins en énergie solaire et selon l'occupation du bâtiment. La proposition de De Luca [61] se distingue par la recherche d'un temps de coupure selon chaque orientation de façade. Le chercheur a utilisé le principe de Pareto pour délimiter le temps de coupure dans une démarche d'optimisation multi-objectif. Le premier objectif dérive de la norme estonienne qui exige un taux d'éclairage de $50 \%$. Le deuxième est de maximiser le volume de l'enveloppe solaire. Le troisième est de maximiser la somme des heures minimales d'ensoleillement direct. De Luca et al. [48] ont étudié différentes ordonnances du temps de coupure d'accès solaire: 1- pendant des heures spécifiques ; 2- pour une quantité d'heures précise et 3- selon un pourcentage du temps d'accès solaire. L'approche offre la possibilité de sélectionner la quantité et la qualité des heures d'accès solaire.

Le temps de coupure était déterminé aussi selon le confort thermique de l'espace urbain [24] ou celui de l'espace intérieur [25]. Pereira et al. [8] ont mis au point une méthode pour calculer le temps de coupure pour différentes orientations selon les exigences de la psychophysiologie humaine. La méthode permet de déterminer les heures de rayonnement indésirable et désirable et de calculer le temps d'ensoleillement utile.

La deuxième contrainte est la clôture d'ombre, délimitée initialement par la base des façades des constructions voisines recevant de l'ensoleillement. L'environnement avoisinant influence le traçage de la clôture d'ombre. Les besoins d'accès solaire d'un hangar ou d'un parc ne sont pas les mêmes besoins que ceux d'un espace d'habitation ou de commerce. C'est pourquoi des recherches ont rehaussé la clôture d'ombre si les locaux de

\footnotetext{
4 L'outil a été développé par le CERMA. Il permet de calculer le flux solaire reçu par une surface pour une orientation, une latitude, une date et une heure données.

${ }^{5}$ La loi de Pareto décrit un phénomène empirique constaté dans certains domaines : environ $80 \%$ des effets sont le produit de $20 \%$ des causes (Pareto, 1965).
} 
rez-de-chaussée des bâtiments voisins n'ont pas besoin d'accès solaire [7, 12, 62]. Bruce [60] a proposé d'affiner la contrainte de clôture d'ombre selon le mode d'occupation des constructions voisines. Il est possible aussi de prendre en considération l'ensoleillement des voiries et des trottoirs [17].

La clôture d'ombre en tant que limite géométrique est une simplification de la réalité urbaine. Elle a été un frein pour son développement. Le polygone de clôture d'ombre a été dématérialisé pour inclure des zones de la façade ou les ouvertures du bâtiment recevant l'ensoleillement [53]. L'outil informatique a permis de mieux gérer la complexité de la forme urbaine. Le traitement par nuage de points [63-65] a permis d'intégrer de manière plus précise les formes complexes des bâtisses voisines pour la génération de l'enveloppe solaire.

\subsection{Dérivées de l'enveloppe solaire}

L'évolution du numérique a permis d'inclure des contraintes physiques et réglementaires à l'enveloppe solaire et de développer des enveloppes dites dérivées (Tableau 1).Nous proposons de classer les enveloppes dérivées en quatre groupes.

Le premier groupe d'enveloppes ajoute à l'enveloppe solaire un objectif de captage solaire. Capeluto et al. [47] ont proposé le Volume Solaire (Solar Volume) permettant l'accès solaire aux constructions voisines ainsi que celle projetée. Il est compris entre deux enveloppes : l'enveloppe de droit solaire (Solar Right Envelope : SRE) et l'enveloppe de captage solaire (The Solar Collection Envelope : SCE). La première est l'équivalent de l'enveloppe solaire définie par Knowles. La seconde est la surface minimale exposée au soleil pendant une période donnée de l'hiver. Plotnikov et al. [66] ont proposé de générer ce le volume de droit solaire à l'aide d'un outil de modélisation paramétrique. Ratti et al. [22] ont proposé les Iso-Surfaces solaires (Iso-Solar Surfaces). Elles correspondent à des surfaces tridimensionnelles des voxels recevant une certaine quantité de rayonnement solaire durant la période choisie. De Luca et al. [67] ont proposé la Multi-iso-surface de collection solaire (Solar Collection Multi-iso-surface) qui permet de passer de l'iso-surface solaire unique introduite par $[9,68]$ en plusieurs iso-surfaces calculées à partir de plans verticaux. Martin et al. [12] ont proposé l'enveloppe solaire passive parfaite (Perfect Passive Solar envelope) qui se base sur une analogie avec la forêt où différents besoins peuvent coexister. Elle correspond à l'intersection de l'enveloppe solaire avec l'extrusion du volume de la parcelle non ombragée par son voisinage. Le volume permet de déterminer l'emplacement des installations de captage solaire.

Le deuxième groupe couple l'enveloppe solaire à des exigences d'accès solaire pour les espaces extérieurs en intégrant la notion d'éventail solaire (Solar Fan) [69]. Camporeale [70] a proposé un modèle qui inclut une préoccupation d'accès solaire aux espaces ouverts à l'échelle du parcellaire. Vartholomaios [58] a proposé l'enveloppe du bloc solaire résidentiel (Residential Solar Block Envelope) qui combine le concept de l'enveloppe solaire et la notion de Bloc Solaire Résidentiel introduit par Okeil [71].

Le troisième groupe introduit des paramètres physiques à l'enveloppe solaire. Dekay [72] a proposé l'Enveloppe Climatique (Climatic Envelope) qui combine l'enveloppe d'éclairage naturel (Daylight Envelope) et l'enveloppe solaire. Saleh [73] a proposé l'Enveloppe paramétrique de confort urbain (Parametric Urban Confort Enveloppe). Il s'agit d'un volume assurant l'accès solaire et la ventilation naturelle de l'espace urbain. Knowles et al. [74] ont proposé l' Interstitium comme limite entre l'enveloppe d'hiver et l'enveloppe d'été. Il s'agit d'une enveloppe adaptative aux changements saisonniers, aux besoins d'accès solaire et aux demandes énergétiques selon la saison. Raboudi et al. [57] ont proposé l'enveloppe de contrôle solaire conditionné à la fois par les règles de l'enveloppe solaire et les règles de l'enveloppe d'ombrage. Celle-ci est le volume minimal 
permettant de garantir l'ombrage des constructions pendant les périodes de surchauffe en été.

Le quatrième groupe ajoute un paramètre règlementaire à l'enveloppe solaire. Raboudi et al. [75] ont proposé le Volume Englobant Solaire. Le modèle combine le volume englobant réglementaire [76] traduisant les règles urbaines et l'enveloppe solaire. Betti et al. [49] ont proposé l'enveloppe solaire graduée (Graded solar envelope) qui tient compte des exigences réglementaires et des exigences d'accès solaire. La recherche a été expérimentée dans le cadre d'un concours d'architecture chinois, où des réglementations strictes en matière d'accès solaire étaient appliquées. Raboudi [77] et Koubaa Turki [43] ont proposé des générateurs de règles urbaines à partir d'enveloppes dérivées de l'enveloppe solaire.

Tableau 1.Présentation des enveloppes dérivées selon la contrainte ajoutée

\begin{tabular}{|c|c|c|c|c|c|c|c|c|c|c|c|c|c|c|c|c|}
\hline & Nom de l'enveloppe en anglais & 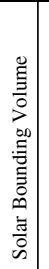 & 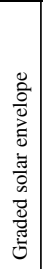 & 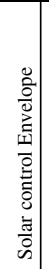 & 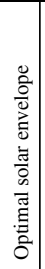 & 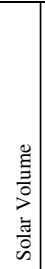 & 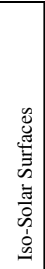 & 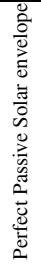 & 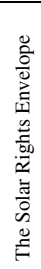 & 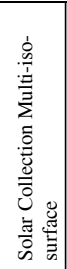 & 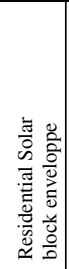 & 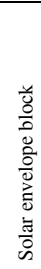 & 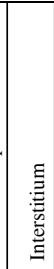 & 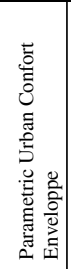 & 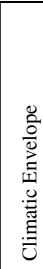 & है \\
\hline & Date de parution & $\overrightarrow{\bar{\Xi}}$ & $\stackrel{ }{\vec{\partial}}$ & $\stackrel{ }{\vec{D}}$ & ્ָડ & $\hat{\sigma}$ & ஜ̊ & હ્ઠ & $\stackrel{\circ}{\stackrel{2}{\sim}}$ & 호 & $\stackrel{n}{\stackrel{2}{2}}$ & $\stackrel{m}{\stackrel{2}{(}}$ & ণ্ণ & 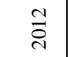 & હ্ণ & \\
\hline \multirow{7}{*}{ } & Intégration de règles urbaines & 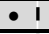 & $\bullet$ & $\mathbf{I}$ & I & I & 1 & & & 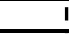 & I & 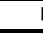 & $\mathbf{I}$ & 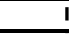 & & 2 \\
\hline & Génération de règles urbaines & $T$ & & $\bullet$ & $\bullet 1$ & T & 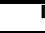 & & & 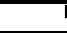 & $\bar{T}$ & 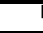 & $\mathbf{T}$ & 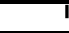 & T & 2 \\
\hline & Captage solaire & & & & & $\bullet$ & $\bullet$ & 0 & $\bullet$ & $\bullet$ & $\bullet$ & & & & & 6 \\
\hline & Accès solaire des espaces extérieurs & & & & & & & & & & $\bullet$ & $\bullet$ & & & & 2 \\
\hline & Ventilation naturelle & I & 1 & 1 & I & I & 1 & & & 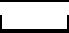 & 1 & 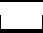 & I. & $\bullet$ & 1 & 2 \\
\hline & Ombrage & $I$ & & 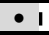 & I & 1 & , & & & $I$ & 1 & 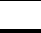 & $\mathbf{I}$ & 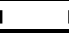 & $\mathbf{I}$ & 1 \\
\hline & Eclairage naturel & I & T & $I$ & 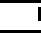 & I & $\mathrm{I}$ & & & & I & 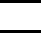 & $I$ & 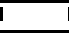 & I & 1 \\
\hline
\end{tabular}

\section{L'enveloppe solaire entre ambitions et réalité}

Nous relevons 118 publications depuis 1980. Leur répartition décennale successive est de $7,12,33$ et 66 . Nous constatons un effort de recherche autour du concept de l'enveloppe solaire et une progression significative sur les deux dernières décennies La moyenne du nombre de publications double à chaque décennie par rapport à la décennie précédente avec une moyenne de 6,6 publication/an durant les dix dernières années contre une moyenne de 0,7 durant la première période. Ceci indique que ce modèle est toujours d'actualité.

Nous avons répertorié quatre groupes de travaux de recherche : usage de l'enveloppe solaire, méthodes de génération, méthodes de détermination des contraintes et enveloppes dérivées représentant respectivement $41 \%, 28 \%, 15 \%$ et $15 \%$ du nombre total des publications.

La répartition temporelle des publications (Figure 1) montre que la première décennie a porté principalement sur l'usage de l'enveloppe solaire et ses méthodes de génération. Les outils proposés étaient difficiles à comprendre, non accessibles ou demandaient un temps de calcul important. Ceci aurait freiné le développement et la vulgarisation de l'enveloppe solaire.

Les enveloppes dérivées sont apparues pendant la deuxième décennie où elles représentent un quart des publications. L'avancée numérique aurait permis d'augmenter le nombre des travaux autour des méthodes de génération (42\%) pendant cette même période. Les chercheurs proposent des méthodes de génération qui se basent sur des outils classiques de CAO ou de Scripting. L'usage de l'enveloppe solaire garde sensiblement le même intérêt. 
Pendant la troisième décennie, le nombre de publications a pratiquement triplé par rapport à la précédente. L'usage de l'enveloppe solaire restait un sujet principal des recherches (45\% des travaux). Cette période a marqué l'apparition de publications sur les méthodes de détermination des contraintes. Cette nouveauté peut être corrélée à l'avancée des modèles de simulation énergétique durant cette période.

Le nombre de publications a augmenté pendant la quatrième décennie et représente $56 \%$ depuis l'apparition du concept. Nous proposons l'hypothèse qu'on peut corréler ce développement à l'essor du numérique, notamment l'avènement de la programmation visuelle a permis d'augmenter le nombre de travaux. En effet, l'accessibilité du paramétrique par le biais de la programmation visuelle pour les non informaticiens, notamment les architectes, a facilité la manipulation des paramètres de l'enveloppe solaire. Nous soulignons qu'à partir de l'année 2013, les recherches utilisent exclusivement les outils paramétriques pour la génération de l'enveloppe solaire. La simplicité de ces outils a contribué à vulgariser le concept de l'enveloppe solaire dans la communauté des chercheurs sans permettre sa démocratisation dans la pratique architecturale et urbaine.

Les applications restent essentiellement des expérimentations de recherche et de pédagogie avec un faible impact pratique. L'expérimentation pédagogique [27, 78, 79] a permis de sensibiliser les étudiants à la conception durable. Celle de recherche est souvent sur des terrains fictifs avec une grande simplification du site. Siret [80] a constaté déjà en 2011 que la mise en pratique de l'enveloppe solaire reste encore l'impensé de la règlementation urbaine par les rares cas d'application réelle [81, 82]. La situation n'a pas beaucoup changé pendant cette dernière décennie. Le progrès scientifique n'est pas toujours garant d'un bénéfice social et environnemental. Une double maturité, celle du contexte et du concept, serait une condition nécessaire au déploiement social d'une avancée scientifique en architecture.

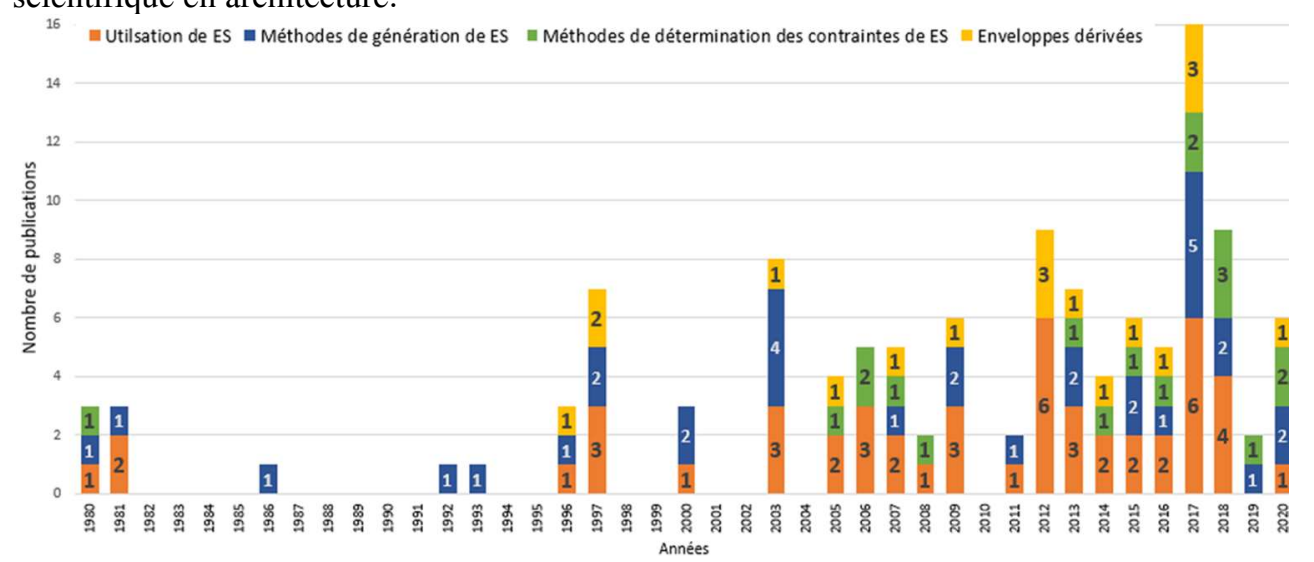

Figure 1.Evolution du nombre de publications sur l'enveloppe solaire de 1980 à 2020

Nous constatons une permanence des travaux de recherche sur les méthodes de génération de l'enveloppe solaire. Elles peuvent être rangées en méthodes par projection et par discrétisation. Les premières projettent l'enveloppe solaire à partir des contraintes [28, 29,50 ] et les secondes se basent sur la subdivision de l'espace de la parcelle en maillage ou en voxel $[25,54]$. La répartition des fréquences de ces méthodes (Figure 2) dans les travaux de 1980 à aujourd'hui selon l'approche numérique utilisée (DAO, scripting, programmation visuelle) montre la montée en puissance de la discrétisation. La rapidité des calculs induite par l'avancée numérique aurait favorisée la discrétisation fine et précise de l'enveloppe, qui est tributaire de la taille de la maille ou du voxel. Cependant, le progrès technique n'est pas garant d'une dissémination sociale de la recherche scientifique en architecture. 


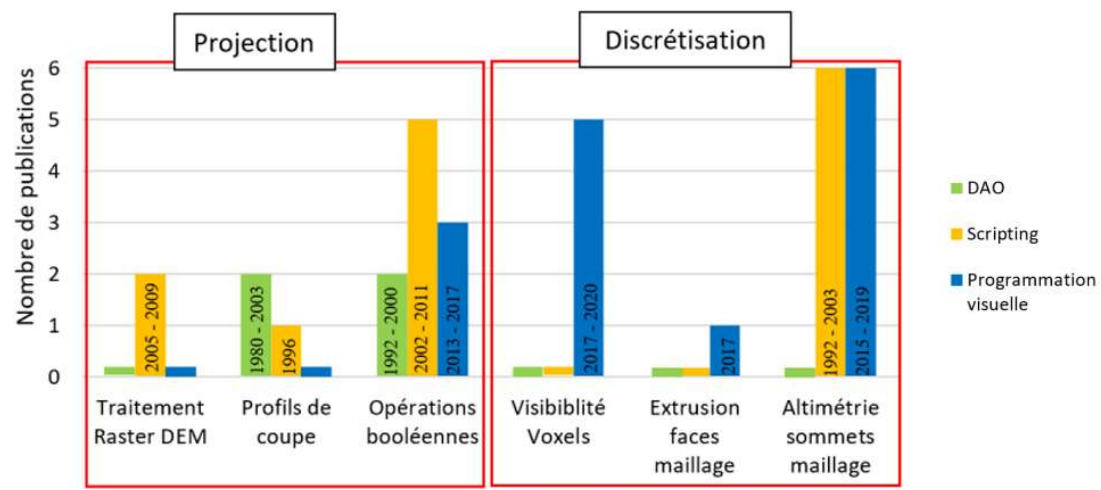

Figure 2.Fréquence des méthodes numériques de génération de l'enveloppe solaire de 1980 à 2020

Un tiers des recherches porte sur la détermination des contraintes de l'enveloppe solaire dont $60 \%$ pour l'étude du temps de coupure et $40 \%$ pour l'étude de la clôture d'ombre. Le captage énergétique est l'objectif principal de 38\% des recherches portant sur la détermination du temps de coupure. Cependant, dans des pays où les mois chauds durent aussi longtemps que les mois froids, il est nécessaire de chercher un équilibre entre l'irradiance solaire reçue en hiver et celle reçue en été [43]. Coupler l'enveloppe solaire à celle d'ombrage [77] est une perspective rendant l'approche opératoire sur toute l'année où la morphologie serait adaptée au changement saisonnier.

L'orientation influence l'ombrage selon le temps de coupure. Or cette période est différente d'une orientation à une autre. Il est utile de proposer des modalités règlementaires qui intègrent les orientations des façades et des heures de références d'irradiance solaire directe. L'objectif serait de favoriser des stratégies de réglementation urbaine en vue d'une meilleure gouvernance de l'espace urbain à l'ère de la ville intelligente. La considération de l'orientation de la surface irradiée offre un instrument de décision précis pour l'établissement de règles urbaines respectant le droit solaire.

Plusieurs chercheurs ont démontré que les ambitions de densification [20,77], de réduction de la consommation énergétique [21] et de préservation du confort thermique [83] que prétend l'enveloppe solaire ne sont pas toujours atteintes. Cependant, la plupart des travaux se focalisent sur l'accès solaire pour un bâtiment isolé sans prendre en considération les rétroactions et la mutualisation avec son environnement immédiat. Nous proposons que la densification et les gains énergétiques méritent d'être affinés par une meilleure définition de ses conditions d'optimisation. Une approche de mutualisation [59] prenant en compte les interactions à l'échelle du voisinage constitue une piste prometteuse. La gestion des différentes interactions et la mutualisation de l'ombrage et de l'accès solaire entre plusieurs parcelles voisines constituent de nouveaux défis pour une morphologie garante de l'accès solaire.

Il serait intéressant de développer un générateur morphologique capable de générer plusieurs volumes interdépendants. Il assisterait le concepteur pour l'établissement d'une maquette numérique interactive. Celle-ci permettrait de garantir à la fois un compromis entre une meilleure densification urbaine et un accès solaire optimal par mutualisation entre les différents volumes appartenant à un ilot urbain.

Cette perspective reste soumise à la nécessité d'une évolution de la culture des pratiques architecturales et urbaines. Les architectes praticiens et urbanistes connaissent-ils les travaux de l'enveloppe solaire ? Ne faut-il pas comme en médecine, mettre une obligation de suivre des formations de mise à jour des connaissances architecturales et urbaines tous les cinq ans ? C'est un moyen de distiller l'effort de recherche et le progrès cognitif dans la pratique. 


\section{Conclusion}

Cet article dresse un inventaire réflexif des approches de l'enveloppe solaire et permet de comprendre l'implication du numérique dans le développement de ce concept et ses méthodes de génération. Actuellement, la modélisation paramétrique et visuelle participe à sa dissémination académique. L'évolution des modèles de simulation solaire et de calculs énergétiques actualise les potentialités de ce modèle pour un développement urbain durable. Cependant, malgré le nombre de travaux consacrés à ce concept, l'usage social et économique des modèles proposés demeurent insuffisant au vu de l'évolution numérique. Cet article en propose ici de nouvelles perspectives pour mieux profiter de l'avancée numérique actuelle et pour répondre au défi climatique actuel.

\section{Bibliographie}

[1] M. Twarowski, Soleil et architecture. Varsovie, Pologne, 1967. 1974 .

[3] R. Gicquel et P. Achard, «European passive solar handbook : basic principles and concepts for passive solar architecture », Brussels, 1986.

[4] N. Baker et K. Steemers, The LT Method 2.0 : An Energy Design Tool for Non-domestic Buildings. Cambridge Architectural Research Limited, 1995.

[5] D. Siret, «Propositions pour une approche déclarative des ambiances dans le projet architectural. Application à l'ensoleillement », Thèse de doctorat, Université de Nantes, 1997.

[6] Littlefair., " Passive solar urban design: ensuring the penetration of solar energy into the city », Renew. Sustain. Energy Rev., 1998.

[7] R. Knowles, «The Solar Envelope », vol. 2 Solar L, p. Rep. 263, 298, 1980.

[8] F. Pereira, C. Silva, et B. Turkienikz, « A methodology for sunlight urban planning : a computerbased solar and sky vault obstruction analysis », Sol. Energy, vol. 70, n 3, p. 217-226, 2001.

[9] A. Marsh, "Computer-Optimised shading design », in Proceedings of IBPSA 2003 Conference, 2003, p. 831-838.

[10] I. G. Capeluto, A. Yezioro, T. Bleiberg, et E. Shaviv, « From computer models to simple design tools : solar rights in the design of urban streets », in Proceedings of IBPSA 2005 Conference, 2005, p. 131-138.

[11] Z. Kristl et A. Krainer, "Site layout as a function of shading in Karst region », in Proceedings of PLEA2005 Conference, 2005, p. 1097-1102.

[12] C. Martin et G. Keeffe, «The Biomimetic Solar City: Solar Derived Urban Form using a Forest growth Inspired Methodology », in Proceedings of PLEA2007 Conference, 2007.

[13] D. Pérez et É. Fávero, «O envelope solar como instrumento deregulamentação do direito de acesso ao Sol : estudode casos na cidade de Campinas SP, Brasil », Vasa, p. 1-15, 2008.

[14] E. Morello, V. Gori, C. Balocco, et C. Ratti, « Sustainable Urban Block Design through Passive Architecture A tool that uses urban geometry optimization to compute energy savings », in Proceedings of PLEA2009 Conference, 2009.

[15] G. Casabianca, J. M. Evans, et S. Schiller, «Solar right and planing codes », in Proceedings of PLEA91 Conference, 1991.

[16] R. Knowles, « The solar envelope: Its meaning for energy and buildings », Energy Build., vol. 35, ${ }^{\circ}$ 1, p. $15-25,2003$.

[17] I. G. Capeluto, A. Yezioro, T. Bleiberg, et E. Shaviv, «Solar Rights in the Design of Urban Spaces ", in Proceedings of PLEA2006 Conference, 2006.

[18] E. Assis, R. Souza, J. Grosch, et L. Ramos, « Integração do conceito de envelope solar na legislação urbana integração do conceito de envelope solar na », in IICBEE 2007, 2007.

[19] K. Raboudi, A. Belkaïd, et A. Ben Saci, "Morphose numérique du volume englobant solaire ", in complexité(s) des modèles de l'architecture numérique. Actes de SCAN'12: Séminaire de Conception Architecturale Numérique, 2012, p. 179-189.

[20] T. N. Stasinopoulos, «A survey of solar envelope properties using solid modelling », J. Green Build., vol. 13, n 1, p. 3-30, 2018.

[21] J. Niemasz, J. Sargent, et C. F. Reinhart, «Solar zoning and energy in detached dwellings », Environ. Plan. B Plan. Des., vol. 40, nº 5, p. 801-813, 2011.

[22] C. Ratti et E. Morello, «SunScapes: extending the 'solar envelopes' concept through 'iso-solar surfaces' ", in Proceedings of PLEA2005 Conference, 2005.

[23] P. Santiago, «Otimização Evolutiva De Forma Para Sustentabilidade , Cruzando O Bim E a Modelação ", in $1^{\circ}$ Congresso Português de Building Information Modelling, 2016.

[24] T. Sorayaei et Z. Sorayaei, «An Integrated approach to Climate Conscious Urban Design Using Solar Envelope Concept », Palma J., vol. 16, n 3, p. 322-330, 2017. 
[25] I. G. Capeluto et B. Plotnikov, « A method for the generation of climate-based, context-dependent parametric solar envelopes », Archit. Sci. Rev., vol. 60, nº 5, p. 395-407, 2017.

[26] A. Vonderohe et A. Asce, « Geometry of Solar Envelopes », J. Surv. Eng., vol. 112, n 1, 1986.

[27] K. Coleman et R. Knowles, « A case study in design studio teaching: the parallel Studio », in The Design Studio in the 21st Century: Proceedings of the 1992 ACSA West Regional Meeting., 1992.

[28] J. F. Cotton, «Solid modeling as a tool for constructing Solar Envelopes », Autom. Constr., vol. 5, no 3, p. 185-192.

[29] T. N. Stasinopoulos, "Solar Envelope - A construction method using AutoCAD 2000 », http://oikotekton.eu/ solenvelope., 2000.

[30] K. Kensek et R. Knowles, «Solar Access Zoning: Computer Generation of the Solar Envelope », Soldiers, $\mathrm{n}^{\circ} 213,1997$. 1992.

[31] P. Yeh, «Computer Aided Solar Envelope Design. », Thesis, University Of Southern California,

[32] D. Noble et K. Kensek, « Computer generated solar envelopes in architecture », J. Archit., vol. 3, n 2, p. 117-127, 1998.

[33] E. Shaviv, « Design tools for bio-climatic and passive solar buildings », vol. 67, p. 189-204, 2000.

[34] M. Juyal, K. Kensek, et R. Knowles, « SolCAD : 3D Spatial Design Tool Tool to Generate Solar Envelope », in Proceedings of ACADIA 2003 Conference, 2003, p. 410-417.

[35] Z. Kristl et A. Krainer, «PIRAMIDA The solar envelope tool », in Proceedings TIA, 2007.

[36] S. Houpert, «Approche inverse pour la résolution de contraintes solaires et visuelles dans le projet architectural et urbain développement et application du logiciel SVR », Thèse de doctorat, Université de Nantes, 2003

[37] P. Grazziotin, C. Dal, S. Freitas, et F. Pereira, « Visualization Techniques in a Building Potential Simulator Using Sunlight Access Control », in proceedings of SIACG, 2002.

[38] K. Kensek, « Solar Access Zoning + Building Information Modeling », p. 1-8, 2013.

[39] M. S. Roudsari, M. Pak, A. Smith, et G. Gill, « Ladybug: a parametric environmental plugin for grasshopper to help designers create an environmentally-conscious design ", in Proceedings of IBPSA 2013 Conference, 2013.

[40] B. Plotnikov, « New Solar Envelope Component now available. », 2015. [En ligne]. Disponible sur: http://www.grasshopper3d.com/group/ladybug/ forum/topics/new-solar-envelope-component-now-available.

[41] J. A. Jakubiec et C. F. Reinhart, «DIVA 2.0 : integrating daylight and thermal simulations using Rhinoceros 3D, Daysim and Energyplus ", in Proceedings of IBPSA 2011 Conference, 2011.

[42] T. Leduc et K. Hartwell, «Limiting the buildings'envelopes in order to prevent the surrounding mask effect : towards an efficient implementation in the context of SketchUp », in Proceedings of PLEA2017 Conference, 2017, p. 2077-2083.

[43] L. Koubaa Turki, "Modèle paramétrique d'optimisation des contraintes de l'enveloppe solaire », Thèse de doctorat, Université de Carthage, 2020.

[44] R. Knowles, Sun Rhythm Form. Cambridge: MIT Press, 1981.

[45] B. Topaloğluin, "Solar Envelope And Form Generation In Architecture. », Master thesis, The Middle East Technical University, 2003.

[46] M. Schiller et U.-F. P. Yeh, «Solvelope: An interactive Computer Program for defining and drawing solar envelopes ", in Proceedings ASESC conference, 1993.

[47] I. G. Capeluto et E. Shaviv, « Modeling the design of urban fabric with solar rights considerations », in Proceedings of IBPSA 1997 Conference, 1997, p. 341-347.

[48] F. De Luca et T. Dogan, «A novel solar envelope method based on solar ordinances for urban planning », Build. Simul., vol. 12, n 5, p. 817-834, 2019.

[49] G. Betti et S. Arrighi, «A differential growth approach to solar envelope generation in complex urban environments », in Proceedings of PLEA2017 Conference, 2017.

[50] E. Morello et C. Ratti, « Sunscapes : "Solar envelopes" and the analysis of urban DEMs ", Comput. Environ. Urban Syst., vol. 33, n 1, p. 26-34, 2009.

[51] A. Jain, K. Kensek, T. Spiegelhalter, M. Schiler, F. Banaei-kashani, et D. Noble, « Development and Validation of a U-BIM Model For mitigation of the urban heat island effect ", in Proceedings of PLEA2009 Conference, 2009.

[52] Z. Kristi et A. Krainer, « Determination of influential area with solar envelope », Gradb. Vestn., vol. 56, p. 156-163, 2007.

[53] F. De Luca, "Solar Form-finding . Subtractive Solar Envelope and Integrated Solar Collection Computational Method for High-rise Buildings in Urban Environments ", in Proceedings of ACADIA 2017 Conference, 2017, p. 212-221.

[54] I. Darmon, "Voxel computational morphogenesis in urban context: proposition and analysis of rules-based generative algorithms considering solar access », 2018.

[55] G. J. Ward, "The Radiance Lighting Simulation and Rendering System », in Proceedings of SIGGRAPH Conference, 1994, p. 459-472.

[56] D. Crawley et al., « EnergyPlus : creating a new-generation building energy simulation program », Energy Build., vol. 33, n 4, p. 319-331, 2001. 
[57] K. Raboudi et A. Ben Saci, «Génération de volumes de contrôle solaire », in Interaction(s) des maquettes numériques: Acte du 6ème Séminaire de Conception Architecturale Numérique. Actes de SCAN'14, 2014, p. 221-231.

[58] A. Vartholomaios, «The residential solar block envelope: A method for enabling the development of compact urban blocks with high passive solar potential », Energy Build., vol. 99, p. 303-312, juill. 2015.

[59] L. Koubaa Turki, K. Raboudi, et A. Ben Saci, «Stratégies de prospect du droit solaire par l'immersion ", in Immersion - émersion et conception de projets architecturaux et urbains : Actes du 8ème Séminaire de Conception Architecturale Numérique. Actes de SCAN 18, 2018, vol. 47, p. 1-11.

[60] G. Bruce, «High Density, Low Energy: Achieving useful solar access for Dublin's multi-storey apartment developments », in proceedings of PLEA2008 conference, 2008.

[61] F. De Luca, "Solar Envelope Optimization Method for Complex Urban Environments », in Proceedings of CAADence in Architecture Conference, 2016, p. 195-201.

[62] K. Raboudi et A. Ben Saci, «A morphological generator of urban rules of solar control», in Proceedings of PLEA2013 Conference, 2013.

[63] M. F. Alkadri, M. Turrin, et S. Sariyildiz, « The use and potential applications of point clouds in simulation of solar radiation for solar access in Urban contexts », Adv. Comput. Des., vol. 3, nº 4 Special Issue, p. 319-338, 2018.

[64] M. F. Alkadri, F. De Luca, et M. Turrin, « Making use of Point Cloud for Generating Subtractive », vol. 1 , p. 633-640, 2019

[65] M. F. Alkadri, F. De Luca, M. Turrin, et S. Sariyildiz, « An integrated approach to subtractive solar envelopes based on attribute information from point cloud data », Renew. Sustain. Energy Rev., vol. 123, 2020.

[66] B. Plotnikov, G. Schubert, et F. Petzold, «Tangible Grasshopper: A method to combine physical models with generative, parametric tools ", in eCAADe 2016, 2016, vol. 2, p. 127-136.

[67] F. De Luca et H. Voll, « Solar Collection Multi-isosurface Method Computational Design Advanced Method for the Prediction of Direct Solar Access in Urban Environments ", in Proceedings of CAAD Futures Conference, 2017, vol. 724.

[68] C. Ratti et E. Morello, «SunScapes : extending the 'solar envelopes' concept through 'iso-solar surfaces'», Proc. PLEA2005 Conf., 2005.

[69] P. Bosselmann, J. Flores, et T. O’Hare, «Sun and Light for Downtown San Francisco », O’Hare, Terrence, 1983.

[70] P. Camporeale, « Genetic Algorithms Applied to Urban Growth Optimization: Solar envelope and solar fan », in eCAADe 2013, 2013.

[71] A. Okeil, «A Holistic approach to energy efficient building forms », Energy Build., vol. 42, p. 1437-1444, 2010.

[72] M. Dekay, T. Moir-McClean, et G. V. Studio, « Greencenter planning for environmental quality in downtown Chattanooga », 2003

[73] M. Saleh, «Using the Tools of Parametric Urbanism Toward a more Responsive Environmental Urban Morphology », Master thesis, University of Alexandria, 2012.

[74] R. Knowles et K. Kensek., "The interstitium: a zoning strategy for seasonally adaptive architecture ", in Proceedings of PLEA2000 Conference, 2000.

[75] K. Raboudi, A. Belkaïd, et A. Ben Saci, "Système multi agents pour la modélisation du volume englobant solaire », Proc. Biskra Archit. Sustain. Int. Conf., 2011.

[76] A. Belkaid et A. Ben Saci, «Approche multi-agents de modélisation du volume englobant réglementaire ", in Conception architecturale numérique et approches environnementales. Séminaire de Conception Architecturale Numérique. Actes de SCAN 09, 2009, p. 121-134.

[77] K. Raboudi, « Générateur morphologique de règles urbaines de contrôle solaire », Thèse de doctorat, Université de Carthage., 2017.

[78] F. Canan et B. Tosunlar, «The Implementation of Sustainable Approaches in the Architectural Design Studio Developing Architectural Designs Using the Solar Envelope Method », ICONARP Int. J. Archit. Plan., vol. 4, n 1 , p. 14-33, 2016.

[79] K. Macháčová, J. Keppl, et L. Krajcsovics, «the Solar Envelope Method in Education At the Faculty of Architecture Stu Bratislava », Proc. CESB 2013 Conf., p. 1-4, 2013.

[80] D. Siret, «Les enveloppes solaires de Ralph Knowles, ou les ambivalences d'un modèle de régulation des formes urbaines », Lieux Communs, Cah. du LAUA, vol. 14, p. 195-206, 2011.

[81] R. Knowles, «On being the right size: An Urban Housing Study for Los Angeles », 1999. [En ligne]. Disponible sur: http://www-bcf.usc.edu/ rknowles/right_size/right_size.html. [Consulté le: 04-juill-2018].

[82] E. Shaviv, A. Yezioro, et I. Capeluto, "Sun and wind in a new businessdistrict in Tel Aviv », in Proceedings of IBPSA 2001 Conference, 2001, p. 151-158.

[83] B. Paramita et M. D. Koerniawan, "Solar envelope assessment in tropical region building case study: Vertical settlement in Bandung, Indonesia », in Proceedings of SUSTAIN 2012 Conference, 2012, vol. 17, p. $757-766$. 